International Journal of Pure and Applied Mathematics

Volume 95 No. 2 2014, 209-221

ISSN: 1311-8080 (printed version); ISSN: 1314-3395 (on-line version)

url: http://www.ijpam.eu

doi: http://dx.doi.org/10.12732/ijpam.v95i2.7

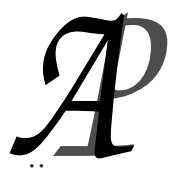

ijpam.eu

\title{
POSTULATION OF DOUBLE POINTS WITH RESTRICTED SUPPORT: THE CASE OF A SMOOTH QUADRIC 3-FOLD
}

\author{
E. Ballico \\ Department of Mathematics \\ University of Trento \\ 38123 Povo (Trento) - Via Sommarive, 14, ITALY
}

\begin{abstract}
Let $D$ be an integral hypersurface of the projective variety, $D \subset X$ a hypersurface and $L$ a line bundle on $X$. Let $E_{x, y} \subset X$ be a general union of $x$ double points of $X$ and $y$ double points of $X$. We classifies the triple $(L, x, y)$ for which $h^{1}\left(\mathcal{I}_{E_{x, y}} \otimes L\right) \cdot h^{0}\left(\mathcal{I}_{E_{x, y}} \otimes L\right)>0$ when $X$ is a smooth quadric 3 -fold and $D$ is a hyperplane section of $X$ and in some cases with $X$ an integral quadric surface.
\end{abstract}

AMS Subject Classification: 14N05, 15A69

Key Words: quadric 3-fold; interpolation, double point, zero-dimensional scheme, secant variety

\section{Introduction}

For any irreducible and projective variety $X$ and any $P \in X_{\text {reg }}$ let $(2 P, X)$ denote the closed subscheme of $X$ with $\left(\mathcal{I}_{P, X}\right)^{2}$ as its ideal sheaf. The scheme $(2 P, X)$ is a zero-dimensional scheme, $(2 P, X)_{\text {red }}=\{P\}$ and $\operatorname{deg}((2 P, X))=$ $1+\operatorname{dim}(X)$. We say that $(2 P, X)$ is a 2 -point of $X$. For any finite set $S \subset X_{\text {reg }}$ set $(2 S, X):=\cup_{P \in S}(2 P, X)$. We say that $(2 P, X)$ is a 2-point of $X$.

Received: April 21, 2014

(c) 2014 Academic Publications, Ltd. url: www.acadpubl.eu 
Theorem 1. Let $X \subset \mathbb{P}^{4}$ be a smooth quadric hypersurface and let $D \subset Q_{3}$ be an integral hyperplane section of $Q_{3}$. Fix integers $k, x, y$ such that $k \geq 1, x \geq 0$ and $0 \leq 3 y \leq(k+1)^{2}$. Let $Z \subset Q_{3}$ be a general union of $x$ 2-points of $Q_{3}$ and $y$ 2-points of $D$. Then either $h^{0}\left(\mathcal{I}_{Z}(k)\right)=0$ or $h^{1}\left(\mathcal{I}_{Z}(k)\right)=0$, except in the following cases:

(a) $(k, x, y)=(2,3,0)$; we have $h^{0}\left(\mathcal{I}_{Z}(2)\right)=3$ and $h^{1}\left(\mathcal{I}_{Z}(2)\right)=1$.

(b) $(k, x, y)=(2,4,0)$; we have $h^{0}\left(\mathcal{I}_{Z}(2)\right)=1$ and $h^{1}\left(\mathcal{I}_{Z}(2)\right)=3$.

(c) $(k, x, y)=(2,0,3)$; we have $h^{0}\left(\mathcal{I}_{Z}(2)\right)=6$ and $h^{1}\left(\mathcal{I}_{Z}(2)\right)=1$.

(d) $(k, x, y)=(2,3,1)$; we have $h^{0}\left(\mathcal{I}_{Z}(2)\right)=1$ and $h^{1}\left(\mathcal{I}_{Z}(2)\right)=2$.

(e) $(k, x, y)=(2,2,2)$; we have $h^{0}\left(\mathcal{I}_{Z}(2)\right)=1$ and $h^{1}\left(\mathcal{I}_{Z}(2)\right)=1$.

(f) $(k, x, y)=(2,1,3)$; we have $h^{0}\left(\mathcal{I}_{Z}(2)\right)=2$ and $h^{1}\left(\mathcal{I}_{Z}(2)\right)=1$.

Take $X, D, k, x, y$ as in Theorem 1. Let $\phi_{k}: X \rightarrow \mathbb{P}^{r}, r:=\left(\begin{array}{c}k+4 \\ 4\end{array}\right)-\left(\begin{array}{c}k+2 \\ 4\end{array}\right)$, be the embedding of $X$ by the complete linear system $\left|\mathcal{O}_{X}(k)\right|$. By [1] the statement of Theorem 1 says that the join of $x$ copies of $\phi_{k}(X)$ and $y$ copies of $\phi_{k}(D)$ has the expected dimension if and only if $(k, x, y)$ is not one of the exceptional cases listed in Theorem 1.

The case $y=0$ of Theorem 1 is true $([3])$.

We work over an algebraically closed field $\mathbb{K}$ with $\operatorname{char}(\mathbb{K})=0$. We use at several steps this assumption.

\section{Surfaces}

For any line bundle $L$ on $X$, any closed subscheme $Z$ of $X$ and any vector space $V \subseteq H^{0}(X, L)$ set $V(-Z):=V \cap H^{0}\left(X, \mathcal{I}_{Z} \otimes L\right)$. Let $D \subset X$ be an effective Cartier divisor of $X$. For every closed subscheme $Z \subset X$ the residual scheme $\operatorname{Res}_{D}(Z)$ of $Z$ with respect to $D$ is a closed

For every line bundle $L$ on $X$ we have an exact sequence of coherent sheaves:

$$
0 \rightarrow \mathcal{I}_{\operatorname{Res}_{H}(Z)} \otimes L(-D) \rightarrow \mathcal{I}_{Z} \otimes L \rightarrow \mathcal{I}_{Z \cap D, D} \otimes L_{\mid D} \rightarrow 0
$$

From (1) we get the following inequalities

- $h^{0}\left(X, \mathcal{I}_{Z} \otimes L\right) \leq h^{0}\left(X, \mathcal{I}_{\operatorname{Res}_{D}(Z)} \otimes L(-D)\right)+h^{0}\left(D, \mathcal{I}_{Z \cap D, D} \otimes L_{\mid D}\right)$;

- $h^{1}\left(X, \mathcal{I}_{Z} \otimes L\right) \leq h^{1}\left(X, \mathcal{I}_{\operatorname{Res}_{D}(Z)} \otimes L(-D)\right)+h^{1}\left(D, \mathcal{I}_{Z \cap D, D} \otimes L_{\mid D}\right)$. 
We will call "Castelnuovo's inequality " any of these inequalities and call (1) the Castelnuovo's sequence.

Notation 1. When $X$ and $D$ are obvious from the context, $E_{x, y}$ denote a general union of $x$ 2-points of $X$ and $y$ 2-points of $D$. Set $E_{x}:=E_{x, 0}$.

Remark 1. Fix any line bundle $L$ on $X$. The semicontinuity theorem for cohomology gives $h^{0}\left(\mathcal{I}_{E_{x+y}} \otimes L\right) \leq h^{0}\left(\mathcal{I}_{E_{x, y}} \otimes L\right)$.

Lemma 1. Fix a zero-dimensional scheme $Z \subset X$, a line bundle $L$ on $X$ and take a general $P \in X$. Set $n:=\operatorname{dim}(X), a:=h^{0}\left(\mathcal{I}_{Z} \otimes L\right)$ and $b:=$ $h^{0}\left(\mathcal{I}_{Z \cup 2 P} \otimes L\right)$. If $a-b=n+1$, then $h^{0}\left(\mathcal{I}_{Z \cup\{2 P, T\}} \otimes L\right)=a-n$ for every divisor $T$ of $X$ with $P \in T_{\text {reg. Now assume }} b \geq a-n$. Set $G:=\operatorname{Aut}(X)$. Assume that the action of $G$ on $X$ has an open orbit, $U$, and that for every $O \in U$ the stabilizer $G_{O} \subset G$ of $O$ acts with an open orbit $U_{O}$ on the set of all codimension one linear subspaces of the tangent space of $X$ at $O$. Assume $D \cap U \neq \emptyset$ and that for a general $O \in D \cap U$ the tangent space $T_{O} D$ of $D$ at $O$ is contained in $U_{O}$. Then there is $g \in G$ such that $h^{0}\left(\mathcal{I}_{g(Z) \cup\{2 O, D\}} \otimes L\right)=b$ for a general $O \in D$.

Proof. Only the last assertion is not obvious. Assume $b \geq a-n$. Hence there is a degree $n$ subscheme $W \subset 2 P$ such that $h^{0}\left(\mathcal{I}_{Z \cup W} \otimes L\right)=b$. Moreover, this is true for an open subset $U_{P}^{\prime}$ of these degree $n$ subscheme. Every degree $n$ subschemes of $2 P$ corresponds to a unique $(n-1)$-dimensional linear subspace of the tangent space of $X$ at $P$ and the converse holds. Hence we may find $h \in G$ with $h(O)=P$ and $h\left(T_{O} D\right)=W$. Use $g:=h^{-1}$.

Lemma 2. Let $T$ be an integral projective surface and $D \subset T$ be an integral Cartier divisor. Fix a zero-dimensional scheme $Z \subset X$, a linear subspace $V$ of $H^{0}(X, L)$ and integers $a \geq 0$ and $b \geq 0$. Let $W \subseteq H^{0}\left(D, L_{\mid D}\right)$ be the image of the restriction map $V(-Z) \rightarrow H^{0}\left(D, L_{\mid D}\right)$. Fix $(a, b) \in \mathbb{N}^{2}$ and let $E \subset D$ be a general union of a 2-points of $D$ and $b$ points of $D$. Set $\alpha:=\operatorname{dim}(V)$ and $\beta:=\operatorname{dim}(W)$.

(i) We have $\operatorname{dim}(V(-(Z \cup E))=\alpha-\min \{\beta, 2 a+b\}$.

(ii) If $\operatorname{dim}(V(-Z))=\operatorname{dim}(V)-\operatorname{deg}(Z)$ and $h^{0}\left(X, \operatorname{Res}_{D}(Z) \otimes L(-D)\right)=0$, then $\operatorname{dim}(V(-(Z \cup E)))=\max \{0, \alpha-2 a-b\}$.

Remark 2. Let $X$ be an integral projective variety, $D$ an integral Cartier divisor of $X, Z$ a closed subscheme of $X, L$ a line bundle on $X$ and $V \subseteq$ $H^{0}(X, L)$ a linear subspace. Set $V(-D):=\{f \in V: f \mid D \equiv 0\}$. Fix an integer $y>0$ and take a general $E \subset D$ with $\sharp(E)=y$. We have $V(-E)=$ 
$\max \{\operatorname{dim}(V(-D)), \operatorname{dim}(V)-y\}$. If $V=H^{0}\left(X, \mathcal{I}_{Z} \otimes L\right)$, then $V(-D)=$ $H^{0}\left(X, \operatorname{Res}_{D}(Z) \otimes L(-D)\right)$.

Proof. Since $\operatorname{dim}(Z \cap D)<\operatorname{dim}(D)$ and $E$ is general in $D$, we have $E \cap Z=$ $\emptyset$ and hence the notation $Z \cup E$ is not ambiguos. Since $\operatorname{char}(K)=0$ and $D$ is integral, we have $\operatorname{dim}(W(-E))=\max \{0, \alpha-\beta-2 a-b\}$ ([8]). Part (ii) follows from part (i), because $\beta=\alpha$ in this case by the residual exact sequence (1).

Corollary 1. Let $T$ be an integral projective surface and $D \subset T$ be an integral Cartier divisor. Fix $L \in \operatorname{Pic}(X)$ and a zero-dimensional scheme $Z \subset X$ such that $h^{1}\left(X, \mathcal{I}_{Z} \otimes L\right)=0$ and $h^{0}\left(\mathcal{I}_{\operatorname{Res}_{D}(Z)} \otimes L(-D)\right)=0$. Set $\alpha:=h^{0}\left(X, \mathcal{I}_{Z} \otimes L\right)-h^{0}\left(\mathcal{I}_{\operatorname{Res}_{D}(Z)} \otimes L(-D)\right)$. Fix integer $a \geq 0, b \geq 0$ and take a general $S \cup S^{\prime} \subset D$ such that $\sharp(S)=a, \sharp\left(S^{\prime}\right)=b$ and $S \cap S^{\prime}=\emptyset$.

Then $h^{0}\left(X, \mathcal{I}_{Z \cup\{2 S, D\} \cup S^{\prime}} \otimes L\right)=\max \{0, \alpha-2 a-b\}$ and $h^{1}\left(X, \mathcal{I}_{Z \cup\{2 S, D\} \cup S^{\prime}} \otimes\right.$ $L)=\max \{0,2 a+b-\alpha\}+h^{1}\left(\mathcal{I}_{Z} \otimes L\right)$.

By our definition of the scheme $E_{x}$ we may always assume $E_{x} \cap D=\emptyset$. Hence as a particular case of Corollary 1 we get the following result (part (d) being obvious).

Proposition 1. Let $X$ be an integral projective surface, $L$ a line bundle on $X$ and $D \subset X$ an effective Cartier divisor of $X$. Fix $(x, y) \in \mathbb{N}^{2}$ such that $2 y \leq \eta$. Set $\alpha:=h^{0}\left(\mathcal{I}_{E_{x}} \otimes L\right)-h^{0}\left(\mathcal{I}_{E_{x}} \otimes L(-D)\right)$ and $\eta:=h^{0}(L)-h^{0}(L(-D)$.

(a) If $h^{1}\left(\mathcal{I}_{E_{x}} \otimes L(-D)\right)=0$ and $2 y \leq \eta$, then $h^{1}\left(\mathcal{I}_{E_{x, y}} \otimes L\right)=0$.

(b) If $h^{0}\left(\mathcal{I}_{E_{x}} \otimes L(-D)\right)=0$ and $2 y \leq \eta$, then either $h^{0}\left(\mathcal{I}_{E_{x, y}} \otimes L\right)=0$ or $h^{1}\left(\mathcal{I}_{E_{x, y}} \otimes L\right)=0$.

(c) If $h^{0}\left(\mathcal{I}_{E_{x}} \otimes L\right)=0$, then $h^{0}\left(\mathcal{I}_{E_{x, y}} \otimes L\right)=0$.

(d) We have $h^{1}\left(\mathcal{I}_{E_{x, y}} \otimes L\right)=h^{1}\left(\mathcal{I}_{E_{x}} \otimes L\right)+\max \{2 y-\alpha, 0\}$ and $h^{0}\left(\mathcal{I}_{E_{x, y}} \otimes L\right)=$ $h^{0}\left(\mathcal{I}_{E_{x}} \otimes L\right)-\min \{\alpha, 2 y\}$.

The integer $\eta$ in the statement of Proposition 1 is the image of the restriction $\operatorname{map} H^{0}(L) \rightarrow H^{0}\left(D, L_{\mid D}\right)$.

Proposition 2. Let $X \subset \mathbb{P}^{3}$ be an integral quadric surface and let $D \subset$ $X$ be a smooth hyperplane section. Fix $(k, x, y) \in \mathbb{N}^{3}$ such that $k \geq 1$ and $2 y \leq 2 k+1$, i.e. $y \leq k$. Let $Z \subset X$ be a general union of $x$ 2-points of $X$ and $y$ 2-points of $D$. Then either $h^{0}\left(\mathcal{I}_{Z}(k)\right)=0$ or $h^{1}\left(\mathcal{I}_{Z}(k)\right)=0$, unless $(k, x, y)=(2,3,0)$. If $(k, x, y)=(2,3,0)$, then $h^{0}\left(\mathcal{I}_{Z}(2)\right)=h^{1}\left(\mathcal{I}_{Z}(2)\right)=1$. 
Proof. Set $\epsilon:=3 x+2 y-(k+1)^{2}$. Increasing or decreasing $x$ (for a fixed $y$ ) we may assume $-2 \leq \epsilon \leq 2$ (unless we landle in an exceptional case). If $k=1$, then it is sufficient to notice that $h^{1}\left(\mathcal{I}_{E_{0,0}}(1)\right)=h^{1}\left(\mathcal{I}_{E_{0,1}}(1)\right)=h^{1}\left(\mathcal{I}_{E_{1,0}}(1)\right)=0$ and $h^{0}\left(\mathcal{I}_{E_{2,0}}(1)\right)=h^{0}\left(\mathcal{I}_{E_{1,1}}(1)\right)=0$.

(a) First assume $k=2$. We have $0 \leq y \leq 2$.

(a1) Assume $y=0$. First assume $x=3$. Let $B \subset X$ be a general subset with cardinality 3 . Since $B$ is contained in a unique $C \in\left|\mathcal{O}_{X}(1)\right|$, obviously $h^{0}\left(\mathcal{I}_{E_{3}}(2)\right)>0$. The uniqueness of $C$ and [7] easily implies $h^{0}\left(\mathcal{I}_{E_{3}}(2)\right)=$ 1. Hence $h^{1}\left(\mathcal{I}_{E_{3}}(2)\right)=1$. We also get $h^{1}\left(\mathcal{I}_{E_{x}}(2)\right)=0$ for all $x \leq 2$ and $h^{0}\left(\mathcal{I}_{E_{x}}(3)\right)=0$ for all $x \geq 4$.

(a2) Assume $y>0$. Since $h^{0}\left(\mathcal{I}_{E_{3}}(2)\right)=1$, we have $h^{0}\left(\mathcal{I}_{E_{3, y}}(2)\right)=0$ for all $y>0$. Let $B \subset X$ be a general union of two 2-points. Let $V$ be the image of the restriction map $H^{0}\left(\mathcal{I}_{B}(2)\right) \rightarrow H^{0}\left(D, \mathcal{O}_{D}(2)\right)$. Since $h^{0}\left(\mathcal{I}_{B}(2)\right)=0$ and $h^{0}\left(\mathcal{I}_{B}(3)\right)=3$ (step (a1)), we have $\operatorname{dim}(V)=3$. Lemma 2 (i.e. [8]) gives $h^{0}\left(\mathcal{I}_{E_{2,1}}(2)\right)=1$ (i.e. $h^{1}\left(\mathcal{I}_{E_{2,1}}(2)\right)=0$ and $h^{0}\left(\mathcal{I}_{E_{2,2}}(2)\right)=0$. Fix a general 2-point $A$ of $X$ and a general $A \subset D$ with $\sharp(E)=y$. Since $y \leq 2$, we have $h^{1}\left(D, \mathcal{I}_{\{2 E, D\}}(2)\right)=0$. Since $h^{1}\left(\mathcal{I}_{A}(1)\right)=0$, the Castelnuovo's inequality gives $h^{1}\left(\mathcal{I}_{E_{1, y}}(2)\right)=0$. Taking $\emptyset$ instead of $A$ we get $h^{1}\left(\mathcal{I}_{E_{0, y}}(2)\right)=0$.

(b) Now assume $k \geq 3$. We also assume to have proved the proposition for the line bundle $\mathcal{O}_{X}(k-1)$ and (if $k \neq 3$ ) for the line bundle $\mathcal{O}_{X}(k-2)$. Set $f:=k-y$. Let $A \subset X$ be a general union of $x-f-12$-points. Fix a general $S \cup S^{\prime} \subset D$ such that $\sharp(S)=y, \sharp\left(S^{\prime}\right)=f$ and $S \cap S^{\prime}=\emptyset$. Fix a general $O \in D$. Let $Y$ be a general union of $A \cup 2 S^{\prime} \sup \{2 S, D\}$ and one 2-point of $X$. By the semicontinuity theorem for cohomology ([9], III.12.8), to prove the proposition for the triple $(k, x, y)$ it is sufficient to prove that either $h^{0}\left(\mathcal{I}_{Y}(k)\right)=0$ or $h^{1}\left(\mathcal{I}_{Y}(k)\right)=0$. Since $h^{i}\left(D, \mathcal{I}_{\left\{2\left(S \cup S^{\prime}\right) \cup\{O\}\right.}(k)\right)=0, i=0,1$, by the differential Horace lemma for 2-points ([4], [6], Lemma $5,[5])$, it is sufficient to prove that either $h^{0}\left(\mathcal{I}_{A \cup S^{\prime} \cup\{2 O, D\}}(k-1)\right)=0$ or $h^{1}\left(\mathcal{I}_{A \cup S^{\prime} \cup\{2 O, D\}}(k-1)\right)=0$. Notice that there is no exceptional case $(k, x, y)=(2, x, y)$ with $y>0$. Hence by the inductive assumption either $h^{0}\left(\mathcal{I}_{A \cup\{2 O, D\}}(k-1)\right)=0$ or $h^{1}\left(\mathcal{I}_{A \cup\{2 O, D\}}(k-1)\right)=$ 0 . If $h^{0}\left(\mathcal{I}_{A \cup\{2 O, D\}}(k-1)\right)=0$, then $h^{0}\left(\mathcal{I}_{A \cup S^{\prime} \cup\{2 O, D\}}(k-1)\right)=0$. Hence we may assume $h^{1}\left(\mathcal{I}_{A \cup\{2 O, D\}}(k-1)\right)=0$, i.e. $h^{0}\left(\mathcal{I}_{A \cup S^{\prime} \cup\{2 O, D\}}(k-1)\right)=$ $k^{2}-y-3(x-f-1)-2$ and $h^{0}\left(\mathcal{I}_{A \cup S^{\prime} \cup\{2 O, D\}}(k-1)\right)>0$. If $f=0$, then we are done. Hence we may assume $f>0$. Notice that $\operatorname{deg}(A \cup\{2 O, D\})=k^{2}+\epsilon$; hence if $\epsilon \geq 0$, then our assumption imply $f>\epsilon$. By Remark 2 to check that either $h^{0}\left(\mathcal{I}_{A \cup S^{\prime} \cup\{2 O, D\}}(k-1)\right)=0$ or $h^{1}\left(\mathcal{I}_{A \cup S^{\prime} \cup\{2 O, D\}}(k-1)\right)=0$ it is sufficient to prove that $h^{0}\left(\mathcal{I}_{A}(k-2)\right) \leq \max \left\{0, h^{0}\left(\mathcal{I}_{A \cup S^{\prime} \cup\{2 O, D\}}(k-1)\right)-f\right\}$.

(b1) Assume $k=3$. We have $14 \leq 3 x+2 y \leq 18$ and $y+f=3$. Hence $x-f-1 \geq 2$. Hence $h^{0}\left(\mathcal{I}_{A}(1)\right)=0$, concluding the case $k=3$. 
(b2) Assume $k=4$. We have $3 x+2 y=25+\epsilon$ and $y+f=4$. Hence $3 x-2 f=17+\epsilon$. We have $h^{0}\left(\mathcal{I}_{A \cup\{2 O, D\}}(3)\right)=16-3(x-f-1)-2=17-3 x+3 f$. First assume $x-f \leq 3$. We get $x \geq 11+\epsilon$ and hence $3 x \geq 33+3 \epsilon>25+\epsilon$, a contradiction. If $x-f-1 \geq 4$, then $h^{0}\left(\mathcal{I}_{A}(2)\right)=0$ and hence we are done. Now assume $x-f-1=3$, i.e. $x=9+\epsilon$. Hence $2 y=-2 \epsilon-2$. Hence $\epsilon=-2$, $y=1$ and $x=7$. We first check that $h^{1}\left(\mathcal{I}_{E_{7}}(4)\right)=0$. Take a general union $A^{\prime} \subset X$ of 52 -points. Let $S_{1} \subset D$ be a general subset with $\sharp\left(S_{1}\right)=2$. Step (b1) gives $h^{1}\left(\mathcal{I}_{A^{\prime}}(3)\right)=0$. Since $h^{0}\left(\mathcal{I}_{A^{\prime}}(2)\right)=0$ (step (a)), Remark ++ gives $h^{1}\left(\mathcal{I}_{A^{\prime} \cup S_{1}}(3)\right)=0$. Since $h^{1}\left(D, \mathcal{I}_{\left\{2 S_{1}, D\right\}}(3)\right)=0$ and $\operatorname{Res}_{D}\left(A^{\prime} \cup 2 S_{1}\right)=A^{\prime} \cup S_{1}$, Castelnuovo's inequality gives $h^{1}\left(\mathcal{I}_{A^{\prime} \cup 2 S_{1}}(3)\right) 00$. Fix a general union $F \subset X$ of 7 2-points. We just proved that $h^{1}\left(\mathcal{I}_{F}(3)\right)=0$, i.e. $h^{0}\left(\mathcal{I}_{F}(3)\right)=4$. Let $V \subseteq$ $H^{0}\left(D, \mathcal{O}_{D}(4)\right)$ be the image of the restriction map $H^{0}\left(\mathcal{I}_{F}(4)\right) \rightarrow H^{0}\left(D, \mathcal{O}_{D}(4)\right)$. Since $h^{0}\left(\mathcal{I}_{F}(3)\right)=0(\operatorname{step}(\mathrm{b} 1))$, we have $\operatorname{dim}(V)=4$. Hence $\operatorname{dim}(V(-2 P))=2$ for a general $P \in D([8])$. Hence $h^{1}\left(\mathcal{I}_{E_{7,1}}(4)\right)=0$, concluding the case $k=4$.

(b3) Now assume $k \geq 5$. The inductive assumption gives that either $h^{0}\left(\mathcal{I}_{A}(k-2)\right)=0$ or $h^{1}\left(\mathcal{I}_{A}(k-2)\right)=0$. In the latter case we have $h^{0}\left(\mathcal{I}_{A}(k-\right.$ $2))=(k-1)^{2}-3(x-f-1)$. Hence it is sufficient to check that $f+2 \leq$ $k^{2}-(k-1)^{2}=2 k-1$, i.e. $f \leq 2 k-3$. This inequality is true, because $y+f=k$.

Proposition 3. Fix non-negative integers $u, v, a, b, x, y$ such that $u>0$, $v>0$ and $(a, b) \notin\{(0, c),(c, 0)\}$ for some $c \neq 1$. Assume $2 y \leq \eta$, where $\eta:=(u+1)(v+1)-(u-a+1)(v-b+1)$ if $u \geq a$ and $v \geq b$ and $\eta:=(u+1)(v+1)$ otherwise. Fix an integral $D \in\left|\mathcal{O}_{X}(a, b)\right|$. Then either $h^{0}\left(\mathcal{I}_{E_{x, y}}(u, v)\right)=0$ or $h^{1}\left(\mathcal{I}_{E_{x, y}}(u, v)\right)=0$, except in the following cases:

(a) $y=0, u \equiv v \equiv 0(\bmod 2)$, either $u=2$ or $v=2$, and $x=(u+1)(v+1) / 3$; in this case $h^{0}\left(\mathcal{I}_{E_{x, 0}}(u, v)\right)=h^{1}\left(\mathcal{I}_{E_{x, 0}}(u, v)\right)=1$.

(b) $u=a, b \leq v, 2 x \leq v-b$ and $\eta-x<2 y \leq \eta$; in this case $h^{0}\left(\mathcal{I}_{E_{x, y}}(u, v)\right)=$ $v-b+1-2 x$ and $h^{1}\left(\mathcal{I}_{E_{x, y}}(u, v)\right)=x+2 y-v+b-(u+1)(v+1)$.

(c) $v=b, a \leq u, 2 x \leq u-a$ and $\eta-x<2 y \leq \eta$; in this case $h^{0}\left(\mathcal{I}_{E_{x, y}}(u, v)\right)=$ $u-a+1-2 x$ and $h^{1}\left(\mathcal{I}_{E_{x, y}}(u, v)\right)=x+2 y+u-a-(u+1)(v+1)$.

(d) $u-a \geq 2, v-b \geq 2, u-a \equiv v-b \equiv 0(\bmod 2), x=(u-a+1)(v-b+1) / 3$, $2 y=\eta$; in this case we have $h^{0}\left(\mathcal{I}_{E_{x, y}}(u, v)=h^{1}\left(\mathcal{I}_{E_{x, y}}(u, v)\right)=1\right.$.

Proof. The assumptions on $a$ and $b$ are equivalent to assuming the existence of an integral element of $\left|\mathcal{O}_{X}(a, b)\right|$.The integer $\eta$ is the dimension of the image of the restriction map $H^{0}\left(\mathcal{O}_{X}(u, v)\right) \rightarrow H 0\left(\mathcal{O}_{X}(u, v)\right)$. Hence by [8] we 
may assume $x>0$. If $h^{0}\left(\mathcal{I}_{E_{x, 0}}(u, v)\right)=0$, then $h^{0}\left(\mathcal{I}_{E_{x, y}}(u, v)\right)=0$ for all $y \geq 0$. Hence from now on we assume $h^{0}\left(\mathcal{I}_{E_{x}}(u, v)\right)>0$.

(a) Assume $y=0$. By [2], Proposition 4.1, or [10] or [7] the only exceptional cases are when $u$ and $v$ are even, either $u=2$ or $v=2$ and $x=(u+1)(v+1) / 3$. In this case we have $h^{1}\left(\mathcal{I}_{E_{x}}(u, v)\right)=h^{0}\left(\mathcal{I}_{E_{x}}(u, v)\right)=1$ (e.g. if $u=2$ and $F \subset X$ is a general subset of $X$ with $\sharp(B)=x$ the only element of $\left|\mathcal{I}_{2 B}(2, v)\right|$ is the curve $2 C$, where $C$ is the unique element of $\left|\mathcal{I}_{B}(1, v / 2)\right|$. Since $C \neq D$ (for a general $\left.E_{x}\right)$ we have $h^{0}\left(\mathcal{I}_{E_{x, y}}(u, v)\right)=0$ if $y>0$ and $h^{0}\left(\mathcal{I}_{E_{x}}(u, v)\right)=1$. Hence from now on we assume $y>0$.

(b) Assume $h^{0}\left(\mathcal{I}_{E_{x}}(u-a, v-b)\right)=0$. If $h^{1}\left(\mathcal{I}_{E_{x}}(u, v)\right)=0$, then apply Proposition 1. If $h^{1}\left(\mathcal{I}_{E_{x}}(u, v)\right)>0$ and (as we are assuming) $h^{0}\left(\mathcal{I}_{E_{x}}(u, v)\right)>0$, then step (a) gives $h^{0}\left(\mathcal{I}_{E_{x, y}}(u, v)\right)=0$ for all $y>0$.

(c) By step (b) we may assume $h^{0}\left(\mathcal{I}_{E_{x}}(u-a, v-b)\right)>0, x>0$, and $y>0$. Since $h^{0}\left(\mathcal{I}_{E_{x}}(u-a, v-b)\right)>0$, we have $h^{0}\left(\mathcal{I}_{E_{x, y}}(u, v)\right)>0$. If $h^{1}\left(\mathcal{I}_{E_{x}}(u-a, v-b)\right)=0$, then $h^{1}\left(\mathcal{I}_{E_{x, y}}(u, v)\right)=0$. Hence we may assume $h^{1}\left(\mathcal{I}_{E_{x}}(u-a, v-b)\right)>0$. Since $h^{0}\left(\mathcal{I}_{E_{x}}(u-a, v-b)\right)>0$ and $x>0$, we have $u \geq a, v \geq b$ and $(u, v) \neq(a, b)$. Since $h^{1}\left(\mathcal{I}_{E_{x}}(u-a, v-b)\right)>0$, then either $u \leq a+2$ or $v \leq b+2$. Fix a general union $B \subset X$ of $x$ 2-points of $X$. In particular we have $B \cap D=\emptyset$ and $h^{i}\left(\mathcal{I}_{B}(z, w)\right)=h^{i}\left(\mathcal{I}_{E_{x}}(z, w)\right)$ for all $z, w$. Let $S \subset D$ be a general subset with $\sharp(S)=y$. Let $\alpha$ be the dimension of the image $V$ of the restriction map $H^{0}\left(\mathcal{I}_{B}(u, v)\right) \rightarrow H^{0}\left(D, \mathcal{O}_{D}(u, v)\right)$.

(c1) Assume $u=a$. We have $h^{0}\left(\mathcal{I}_{E_{x}}(0, v-b)\right)>0$ if and only if $2 x \leq$ $v-b$. Assume $2 x \leq v-b$. Since $u>0$, we get $3 x<(u+1)(v+1)$. Hence $h^{1}\left(\mathcal{I}_{E_{x}}(u, v)\right)=0\left(\right.$ step (a)). We have $h^{0}\left(\mathcal{I}_{B}(0, v-b)\right)=v-b+1-2 x$. We have $\eta=(u+1)(b+1)-(v-b+1)$ and $\alpha=\eta-x$. By [8] we have $\operatorname{dim}(V(-2 S))=\max \{0, \alpha-2 y)$. By part (b) of Proposition 1 we get that $h^{1}\left(\mathcal{I}_{E_{x, y}}(u, v)\right)>0$ if and only if $2 y>\eta-x$. Since $h^{1}\left(\mathcal{I}_{E_{x}}(u, v)\right)=0$, we also get that if $2 y>\eta-x$, then $h^{0}\left(\mathcal{I}_{E_{x, y}}(u, v)\right)=v-b+1-2 x$ and $h^{1}\left(\mathcal{I}_{E_{x, y}}(u, v)\right)=$ $x+2 y+v-b+1-(u+1)(v+1)$. In the same way we handle the case $v=b$.

(c2) Assume $u=a+1$. By step (c1) we may assume $v>b$. Assume for the moment that $h^{1}\left(\mathcal{I}_{E_{x}}(u, v)\right)=0$. By part (b) of Proposition 1 we have $h^{0}\left(\mathcal{I}_{E_{x, y}}(u, v)\right) \cdot h^{1}\left(\mathcal{I}_{E_{x, y}}(u, v)\right)=0$, unless $h^{0}\left(\mathcal{I}_{E_{x}}(1, v-b+1)\right)>0$, i.e. unless $3 x \leq 2 v-2 b+3$. Assume $3 x \leq 2 v-2 b+2$. Since $h^{1}\left(\mathcal{I}_{E_{x}}(1, v-b+1)\right)=0$, we get $h^{1}\left(\mathcal{I}_{E_{x, y}}(u, v)\right)=0$. Now assume that $h^{1}\left(\mathcal{I}_{E_{x}}(u, v)\right)>0$. Since $h^{0}\left(\mathcal{I}_{E_{x}}(u, v)\right)>$ 0 and $y>0$, step (a) gives $h^{0}\left(\mathcal{I}_{E_{x, y}}(u, v)\right)=0$.

In the same way we handle the case $v=b+1$.

(c3) Assume $u=a+2$. By steps (c1) and (c2) we may assume $v \geq$ $b+2$. Since $u \geq 3, v \geq 3$ and $h^{0}\left(\mathcal{I}_{E_{x}}(u, v)\right)>0$, we have $h^{1}\left(\mathcal{I}_{E_{x}}(u, v)\right)>$ 0. By part (b) of Proposition 1 we have $h^{0}\left(\mathcal{I}_{E_{x, y}}(u, v)\right) \cdot h^{1}\left(\mathcal{I}_{E_{x, y}}(u, v)\right)=0$, 
unless $h^{0}\left(\mathcal{I}_{E_{x}}(2, v-b)\right)>0$, i.e. unless $x \leq v-b+1$. If $x \leq v-b$, then $h^{1}\left(\mathcal{I}_{E_{x}}(2, v-b)\right)=0$ and hence $h^{1}\left(\mathcal{I}_{E_{x, y}}(u, v)\right)=0$ (Proposition 1). Now assume $x=v-b+1$. If $2 y=\eta$, then $h^{i}\left(D, \mathcal{I}_{\{2 S, D\}}(u, v)\right)=0, i=0,1$, and hence $h^{0}\left(\mathcal{I}_{E_{x, y}}(u, v)\right)=h^{1}\left(\mathcal{I}_{E_{x, y}}(u, v)\right)=1$. Now assume $2 y<\eta$. In this case $\alpha=\eta-1$. By [7] for general $S$ the scheme $\{2 S, D\}$ imposes $2 y$ independent conditions to $V$. Since $h^{1}\left(\mathcal{I}_{E_{x}}(u, v)\right)=0$, we get $h^{1}\left(\mathcal{I}_{E_{x, y}}(u, v)\right)=0$. In the same way we handle the case $v=b+2$.

Proposition 4. Fix integers $k>0, a>0, x \geq 0$ and $y$ such that $0 \leq 2 y \leq \eta$, where $\eta=(k+1)^{2}-(k-a+1)^{2}$ if $a \leq k$ and $\eta:=(k+1)^{2}$ if $a>k$. Let $X \subset \mathbb{P}^{3}$ be an integral quadric cone. Fix an integral curve $D \in\left|\mathcal{O}_{X}(k)\right|$. Then either $h^{0}\left(\mathcal{I}_{E_{x, y}}(k)\right)=0$ or $h^{1}\left(\mathcal{I}_{E_{x, y}}(k)\right)=0$, except if either $(k, x, y)=(2,3,0)$ or $\eta$ is even $k=a+2, x=3$ and $y=\eta / 2$. In these two exceptional cases we have $h^{0}\left(\mathcal{I}_{E_{x, y}}(k)\right)=h^{0}\left(\mathcal{I}_{E_{x, y}}(k)\right)=1$.

Proof. Since $h^{0}\left(\mathcal{O}_{X}(k-a)\right)=0$ if $k<a$ and $h^{0}\left(\mathcal{O}_{X}(k-a)\right)=(k-a+1)$ if $k \geq a$, the integer $\eta$ is the dimension of the image of the restriction map $H^{0}\left(\mathcal{O}_{X}(k)\right) \rightarrow H^{0}\left(D, \mathcal{O}_{D}(k)\right)$. By Proposition 2 we may assume $a \geq 2$ and $y>0$. By Propositions 1 and 2 it is sufficient to study the case $k=a+2$. Assume $k=a+2$. We have $h^{0}\left(\mathcal{I}_{E_{x}}(2)\right)=0$ if and only if $x \geq 4$. Hence we are done if $x \geq 4$ (part (b) of Proposition 1). If $x \leq 2$, then $h^{1}\left(\mathcal{I}_{E_{x}}(2)\right)=0$ and hence $h^{1}\left(\mathcal{I}_{E_{x, y}}(k)\right)=0$ for all $y \leq\lfloor\eta / 2\rfloor$ (part (a) of Proposition 1). Now assume $x=3$. First assume $2 y=\eta$. Since $h^{0}\left(\mathcal{I}_{E_{3}}(2)\right)>0$ and $h^{0}\left(\mathcal{I}_{E_{3}}(2)\right)>0$, we have $h^{0}\left(\mathcal{I}_{E_{x, \eta / 2}}(k)\right)>0$. Notice that $h^{0}\left(\mathcal{I}_{E_{x, \eta / 2}}(k)\right)=h^{1}\left(\mathcal{I}_{E_{x, \eta / 2}}(k)\right)$. Since $h^{1}\left(D, \mathcal{I}_{\{2 S, D\}}(k)\right)=0$ and $h^{1}\left(\mathcal{I}_{B}(2)\right)=h^{1}\left(\mathcal{I}_{E_{3}}(2)\right)=1$, (1) gives $h^{1}\left(\mathcal{I}_{E_{3, \eta / 2}}(a+\right.$ $2)) \leq 1$. Hence $h^{0}\left(\mathcal{I}_{E_{x, \eta / 2}}(k)\right)=h^{1}\left(\mathcal{I}_{E_{x, \eta / 2}}(k)\right)=1$.

Now assume $2 y \leq \eta-2$. Let $B_{1}$ be a general union of two 2-points. Take a general $S \cup S_{1} \subset D$ such that $\sharp(S)=y, \sharp\left(S_{1}\right)=1$ and $S \cap S_{1}=\emptyset$. To prove that $h^{1}\left(\mathcal{I}_{E_{3, y}}(k)\right)=0$ it is sufficient to prove that $h^{1}\left(\mathcal{I}_{B_{1} \cup 2 S_{1} \cup\{2 S, D\}}(k)\right)=0$. Since $\left(B_{1} \cup 2 S_{1} \cup\{2 S, D\}\right) \cap D=\left(2 S_{1} \cup S, D\right\}, \sharp\left(S_{1} \cup S\right) \leq \eta / 2$ and $S_{1} \cup S$ is general in $D$, we have $h^{1}\left(D, \mathcal{I}_{\left\{2\left(S \cup S_{1}\right), D\right\}}(k)\right)=0$. Since $f>0$, we have $x^{\prime} \leq 2$. Hence $h^{1}\left(\mathcal{I}_{B_{1}}(2)\right)=0$. We have $h^{0}\left(\mathcal{I}_{B_{1}}(k-2 a)=0\right.$, because $k-2 a \leq 0$ and $B_{1} \neq \emptyset$. Apply Remark 2 .

Now assume $\eta=2 y+1$. Fix a general $P \in D$. To copy the proof of the case $2 t \leq \eta-2$ it is sufficient to check that $h^{1}\left(\mathcal{I}_{B_{1} \cup\{2 P, D\}}(2)\right)=0$. Hence it is sufficient to prove that $h^{1}\left(\mathcal{I}_{E_{2,1}}(2)\right)=0$. This is true by Proposition 1 , because $h^{0}\left(\mathcal{I}_{E_{2}}(k-a)\right)=0$, 


\section{Proof of Theorem 1}

Proof of Theorem 1. For all integers $k>0$ we have $h^{0}\left(\mathcal{O}_{X}(k)\right)=\left(\begin{array}{c}k+4 \\ 4\end{array}\right)-$ $\left(\begin{array}{c}k+2 \\ 4\end{array}\right)=(k+2)(k+1)(2 k+3) / 6$. Set $\epsilon:=4 x+3 y-(k+2)(k+1)(2 k+3) / 6$. If $k=1$, then it is sufficient to notice that $h^{1}\left(\mathcal{I}_{E_{0,0}}(1)\right)=h^{1}\left(\mathcal{I}_{E_{0,1}}(1)\right)=$ $h^{1}\left(\mathcal{I}_{E_{1,0}}(1)\right)=0$ and $h^{0}\left(\mathcal{I}_{E_{1,1}}(1)\right)=h^{0}\left(\mathcal{I}_{E_{2,0}}(1)\right)=0$. All cases with $y=0$ are known ([3]). Hence we may assume $y>0$. First assume $x=0$. We have $h^{0}\left(\mathcal{I}_{E_{0, y}}(k)\right)>0$ for all $k>1$. Since $3 y \leq(k+1)^{2}$, Proposition 2 gives that $h^{1}\left(\mathcal{I}_{E_{0, y}}(k)\right)=0$ if and only if $k=2$ and $y=3$. Since $h^{i}\left(\mathcal{O}_{X}(1)\right)=0, i=1,2$, we have $h^{1}\left(\mathcal{I}_{E_{0,3}}(2)\right)=1$ and $h^{0}\left(\mathcal{I}_{E_{0,3}}(2)\right)=6$. From now on we assume $x>0$.

(a) Assume $k=2$. First assume $x=3$. Since $h^{1}\left(\mathcal{I}_{E_{x}}(3)\right)>0$ for all $x \geq 3([3])$, we have $h^{1}\left(\mathcal{I}_{Z}(2)\right)>0$ if $x \geq 3$. Since $h^{0}\left(\mathcal{I}_{E_{4}}(2)\right)>0$, Remark 1 gives $h^{0}\left(\mathcal{I}_{E_{3,1}}(2)\right)>0$. Since $h^{0}\left(\mathcal{I}_{E_{3}}(2)\right)=3$ and $h^{0}\left(\mathcal{I}_{E_{4}}(2)\right)=1$, Lemma 1 gives $h^{0}\left(\mathcal{I}_{E_{3,1}}(2)\right)=1$, i.e. $h^{1}\left(\mathcal{I}_{E_{3,1}}(2)\right)=2$. Since $h^{0}\left(\mathcal{I}_{E_{3,1}}(2)\right)=1$ and $h^{0}\left(\mathcal{I}_{E_{3}}(1)\right)=0$, we have $h^{0}\left(\mathcal{I}_{E_{3, y}}(2)\right)=0$ for all $y \geq 2$. Since $h^{0}\left(\mathcal{I}_{E_{4}}(2)\right)=1$, then $h^{0}\left(\mathcal{I}_{Z}(2)\right)=0$ if $x \geq 4$ and $y>0$.

Now assume $x=2$. Since $h^{0}\left(\mathcal{I}_{E_{2}}(1)\right)=0$ and $h^{1}\left(\mathcal{I}_{E_{2}}(2)\right)=0$, the image $V$ of the restriction map $H^{0}\left(\mathcal{I}_{E_{2}}(2)\right) \rightarrow H^{0}\left(D, \mathcal{O}_{D}(2)\right)$ has dimension 8. Since $h^{0}\left(\mathcal{I}_{E_{3}}(2)\right)=3$, Lemma 1 gives $h^{0}\left(\mathcal{I}_{E_{2,1}}(2)\right)=3$, i.e. $h^{1}\left(\mathcal{I}_{E_{2,1}}(2)\right)=0$. Since $h^{0}\left(\mathcal{I}_{E_{4}}(2)\right)=1$, we have $h^{0}\left(\mathcal{I}_{E_{2,2}}(2)\right) \geq 1$ by the semicontinuity theorem. Since $h^{0}\left(\mathcal{O}_{X}(2)\right)=14$, we have $h^{0}\left(\mathcal{I}_{E_{2,2}}(2)\right)=h^{1}\left(\mathcal{I}_{E_{2,2}}(1)\right)$. Assume $h^{0}\left(\mathcal{I}_{E_{2,2}}(2)\right) \geq 2$. Since $h^{0}\left(\mathcal{I}_{E_{2}}(1)\right)=0$ and $h^{0}\left(\mathcal{I}_{E_{2}}(2)\right)=6$, the image $V$ of the restriction map $h^{0}\left(\mathcal{I}_{E_{2}}(2)\right) \rightarrow H^{0}\left(D, \mathcal{O}_{D}(2)\right)$ has dimension 6. Take general $O, P \in D$. Since $h^{0}\left(\mathcal{I}_{E_{2,1}}(2)\right)=3$, we have $\operatorname{dim}(V(-2 O))=3$. To check that $h^{0}\left(\mathcal{I}_{E_{2,2}}(2)\right) \leq 1$ and hence to conclude the case $(x, y)=(2,2)$ it is sufficient to prove that $\operatorname{dim}(V(-2 O-2 P)) \leq 1$. Assume that $\operatorname{dim}(V(-2 O-2 P)) \geq 2$. Since $P$ is general in $D$, it means that the rational map $D \rightarrow \mathbb{P}^{2}$ induced by the linear system $V(-2 O)$ has generically differential zero. Since $\operatorname{dim}(V(-2 O)) \geq 2$ and $\operatorname{char}(\mathbb{K})=0$, this is absurd.

Now assume $x=1$. Sinceh ${ }^{1}\left(\mathcal{I}_{E_{1}}(1)\right)=0$, we get $h^{1}\left(\mathcal{I}_{E_{1, y}}(2)\right)=0$ if $y \leq 2$ and $h^{1}\left(\mathcal{I}_{E_{1,3}}(2)\right) \leq 1$. Now assume $y=3$. Take a general set $S \subset D$ with $\sharp(S)=$ 3. Since $h^{1}\left(D, \mathcal{I}_{\{2 S, D\}}(2)\right)=1, h^{0}\left(\mathcal{I}_{E_{1}}(2)\right)=1$ and $h^{i}\left(\mathcal{I}_{E_{1}}(1)\right)=0, i=1,2$, the Castelnuovo's exact sequence (1) gives $h^{1}\left(\mathcal{I}_{E_{1,3}}(2)\right)=1$ and $h^{0}\left(\mathcal{I}_{E_{1,3}}(2)\right)=2$.

(b) Assume $k>2$ and that Theorem 1 is true for the line bundle $\mathcal{O}_{X}(k-1)$ and, if $k \geq 4$, for the line bundle $\mathcal{O}_{X}(k-2)$. Set $f:=(k+1)^{2}-3 y$ and $g:=(k+1)^{2}-3 y-3 f$. We have $0 \leq g \leq 2$. Let $A \subset X$ be a general union of $x-f-12$-points. Fix a general $S \cup S^{\prime} \cup S^{\prime \prime} \subset D$ such that $\sharp(S)=y, \sharp\left(S^{\prime}\right)=f, \sharp\left(S^{\prime \prime}\right)=g$, and $S \cap S^{\prime}=S \cap S^{\prime \prime}=S^{\prime} \cap S^{\prime \prime}=\emptyset$. Let $Y$ be a general union of $A \cup 2 S^{\prime} \cup\{2 S, D\}$ and $g$ 2-points of $X$. By the 
semicontinuity theorem for cohomology ([9], III.12.8), to prove the proposition for the triple $(k, x, y)$ it is sufficient to prove that either $h^{0}\left(\mathcal{I}_{Y}(k)\right)=0$ or $h^{1}\left(\mathcal{I}_{Y}(k)\right)=0$. Since $h^{i}\left(D, \mathcal{I}_{\left\{2\left(S \cup S^{\prime}\right), D\right\} \cup S^{\prime \prime}}(k)\right)=0, i=0,1$, by the differential Horace lemma for 2-points ([4], [6], Lemma 5, [5]), it is sufficient to prove that either $h^{0}\left(\mathcal{I}_{A \cup S^{\prime} \cup\left\{2 S^{\prime \prime}, D\right\}}(k-1)\right)=0$ or $h^{1}\left(\mathcal{I}_{A \cup S^{\prime} \cup\left\{2 S^{\prime \prime}, D\right\}}(k-1)\right)=0$. If $h^{0}\left(\mathcal{I}_{A \cup\left\{2 S^{\prime \prime}, D\right\}}(k-1)\right)=0$, then $h^{0}\left(\mathcal{I}_{A \cup S \cup\left\{2 S^{\prime \prime}, D\right\}}(k-1)\right)=0$ and hence we are done. Hence we may assume $h^{0}\left(\mathcal{I}_{A \cup\left\{2 S^{\prime \prime}, D\right\}}(k-1)\right)>0$. If $k \geq 4$, then the inductive assumption gives $h^{1}\left(\mathcal{I}_{A \cup\left\{2 S^{\prime \prime}, D\right\}}(k-1)\right)=0$, i.e. $h^{0}\left(\mathcal{I}_{A \cup\left\{2 S^{\prime \prime}, D\right\}}(k-1)\right)=$ $-\epsilon+f$. By Lemma 2 it is sufficient to prove that $h^{0}\left(\mathcal{I}_{A}(k-2)\right) \leq \max \{0,-\epsilon\}$.

(b1) Assume $k=3$. We have $4 x+3 y=30+\epsilon$ and $3 y+3 f+g=16$. Hence $g=1$ and $y+f=5$. Since $h^{0}\left(\mathcal{I}_{E_{7}}(3)\right)=2([3])$ and $h^{0}\left(\mathcal{I}_{E_{8}}(3)\right)=0([3])$, Lemma 1 gives $h^{0}\left(\mathcal{I}_{E_{7,1}}(3)\right)=0$. Since $h^{1}\left(\mathcal{I}_{E_{7}}(3)\right)=0$, we also get $h^{1}\left(\mathcal{I}_{E_{6,1}}(3)\right)=0$. Hence we may assume $y \geq 2$.

(b1.1) First assume $y=2$ and hence $f=3$. It is sufficient to do the case $x=6$. We have $x-f-1=2$. Hence $h^{0}\left(\mathcal{I}_{A}(1)\right)=0$. Since $h^{1}\left(\mathcal{I}_{E_{2,1}}(2)\right)=0$ (step (a)), we have $h^{0}\left(\mathcal{I}_{A \cup\left\{2 S^{\prime \prime}, D\right\}}(2)\right)=3$. Since $h^{0}\left(\mathcal{I}_{A}(1)\right)=0$, Lemma 2 gives $h^{i}\left(\mathcal{I}_{A \cup S^{\prime} \cup\left\{2 S^{\prime \prime}, D\right\}}(2)\right)=0, i=0,1$.

(b1.2) Now assume $y=3$ and hence $f=2$. It is sufficient to do the cases $x=5$ and $x=6$. First assume $x=5$. Since $h^{1}\left(\mathcal{I}_{E_{2,1}}(2)\right)=0(\operatorname{step}(\mathrm{a}))$, we have $h^{1}\left(\mathcal{I}_{A \cup\left\{2 S^{\prime \prime}, D\right\}}(2)\right)=0$. Since $x-f-g=2$, we have $h^{0}\left(\mathcal{I}_{A}(1)\right)=0$. Hence Lemma 2 gives $h^{1}\left(\mathcal{I}_{A \cup S^{\prime} \cup\left\{2 S^{\prime \prime}, D\right\}}(2)\right)=0$. Hence $h^{1}\left(\mathcal{I}_{Y}(3)\right)=0$ and so $h^{1}\left(\mathcal{I}_{E_{5,3}}(3)\right)=0$.

Since $h^{0}\left(\mathcal{I}_{E_{5,3}}(3)\right)=1$, we have $h^{0}\left(\mathcal{I}_{E_{6,3}}(3)\right)=0$.

(b1.3) Now assume $y=4$ and hence $f=1$. It is sufficient to do the cases $x=4$ and $x=5$. First assume $x=4$. By step (a) we have $h^{1}\left(\mathcal{I}_{E_{2,1}}(2)\right)=$ 0 and hence $h^{1}\left(\mathcal{I}_{A \cup\left\{2 S^{\prime \prime}, D\right\}}(2)\right)=0$. Since $h^{0}\left(\mathcal{I}_{A}(1)\right)=0$, Lemma 2 gives $h^{1}\left(\mathcal{I}_{A \cup S^{\prime} \cup\left\{2 S^{\prime \prime}, D\right\}}(2)\right)=0$. Hence $h^{1}\left(\mathcal{I}_{E_{4,4}}(3)\right)=0$. Now assume $x=5$. Since $h^{0}\left(\mathcal{I}_{E_{3,1}}(2)\right)=1$ (step (a)), we have $h^{0}\left(\mathcal{I}_{A \cup\left\{2 S^{\prime \prime}, D\right\}}(2)\right)=1$. Since $x-f-g \geq 2$, we have $h^{0}\left(\mathcal{I}_{A}(1)\right)=0$. Hence Lemma 2 gives $h^{0}\left(\mathcal{I}_{A \cup S^{\prime} \cup\left\{2 S^{\prime \prime}, D\right\}}(2)\right)=0$, i.e. $h^{0}\left(\mathcal{I}_{E_{5,4}}(3)\right)=0$.

(b1.4) Now assume $y=5$. First assume $x=3$. Since $h^{0}\left(\mathcal{I}_{E_{3}}(2)\right)=3([3])$ and $h^{0}\left(D, \mathcal{I}_{\{2 S, D\}}(3)\right)>0$, we have $h^{0}\left(\mathcal{I}_{E_{3,5}}(3)\right) \geq 3$. We have $h^{0}\left(\mathcal{I}_{E_{3,5}}(3)\right)=3$ if and only if $h^{1}\left(\mathcal{I}_{E_{3,5}}(3)\right)=0$. Assume $h^{0}\left(\mathcal{I}_{E_{3,5}}(3)\right) \geq 4$. Let $Z^{\prime} \subset X$ a general union of 32 -points. Let $V \subset H^{0}\left(D, \mathcal{O}_{D}(3)\right)$ the image of the restriction $\operatorname{map} H^{0}\left(\mathcal{I}_{Z^{\prime}}(3)\right) \rightarrow H^{0}\left(D, \mathcal{O}_{D}(3)\right)$. Since $h^{0}\left(\mathcal{I}_{Z^{\prime}}(2)\right)=3$ and $h^{1}\left(\mathcal{I}_{Z^{\prime}}(3)\right)=0$ $([3])$, we have $\operatorname{dim}(V)=15$. Since we assumed that $h^{0}\left(\mathcal{I}_{E_{3,5}}(3)\right) \geq 4$, we have $V(-2 B) \neq 0$ for a general $B \subset D$ with $\sharp(B)=5$, i.e. $V$ is 4-defective in the sense of [7]. We have $D \subset \mathbb{P}^{3}$ and for each curve $C \subset D$ we call $\operatorname{deg}(C)$ the degree of $C$ as a space curve. Let $H \subset D$ be the zero-locus of a general element 
of $V(-2 B)$. Let $\Sigma$ be the union of the positive-dimensional components of $\operatorname{Sing}(H)$ containing at least one of the points of $B$. By [7], $\Sigma \neq \emptyset, B \subset \Sigma$ and for all $P, P^{\prime} \in \Sigma$ the distribution (degrees, number of components and genera) of the components containing $P$ are the same as for the ones containing $P^{\prime}$. We have $H=2 \Sigma+E$ with either $E=\emptyset$ or $E$ an effective curve. Since $\operatorname{deg}(H)=6$, $\Sigma \supset B$ and $B$ is the union of 5 general points, $\Sigma$ must be a rational normal curve contained in $D \subset \mathbb{P}^{3}$ (it cannot be a curve of degree $\leq 2$, a plane curve of degree 3 , the union of 3 lines or the union of a conic and a line). This is impossible if $D$ is a smooth quadric, because in this case we have $H \in\left|\mathcal{O}_{D}(3,3)\right|$ and $H \neq 2 \Sigma$, because 3 is odd. Now assume that $D$ is a quadric cone with vertex $O$ and let $m: F_{2} \rightarrow D$ be its minimal desingularization. The smooth surface $F_{2}$ is a Hirzebruch surface with $h:=m^{-1}(O)$ as its only irreducible curve with negative self-intersection. We have $\operatorname{Pic}\left(F_{2}\right) \cong \mathbb{Z}^{2}$ and we take $h$ and a fiber $F$ of the ruling of $F_{2}$ as a generator of $F_{2}$. Let $\Sigma^{\prime}$ and $H^{\prime}$ denote the strict transform in $F_{2}$ of $\Sigma$ and $H$, respectively. Since $m$ is induced by the complete linear system $|h+2 F|$, we have $\Sigma^{\prime} \in|h+z F|$ for some $z \leq 3$. Since $h^{0}\left(F_{2}, \mathcal{O}_{F_{2}}(h+z F)\right)=2 z$ for all $z>0$, and $\Sigma$ contains 5 general points of $D$, we have $z=3$. We get $\Sigma^{\prime} \in\left|\mathcal{O}_{F_{2}}(h+3 F)\right|$ and that (as we knew) $\Sigma$ is a rational normal curve. Since $h^{0}\left(F_{2}, \mathcal{O}_{F_{2}}(h+3 F)\right)=0$, we also get that $V$ is the vector space associated to the projective space of all $2 C$ with $C$ either a rational normal curve of $D$ (it contains the vertex of $D$ ) or the union of a line and a smooth conic not through the vertex of $D$. In particular $V$ does not depend from the choice of the 3 points of $\left(Z^{\prime}\right)_{\text {red }}$. The projective space $\left|\mathcal{I}_{Z^{\prime}}(3)\right|$ contains the union of an element $T$ of $\left|\mathcal{I}_{Z^{\prime}}(2)\right|$ and a hyperplane section of $X$. Since $h^{0}\left(\mathcal{I}_{Z^{\prime}}(3)\right)=3$, the linear system $\left|\mathcal{I}_{Z^{\prime}}(2)\right|$ is the set of all intersection with $Q$ of the quadric hypersurfaces of $\mathbb{P}^{4}$ whose cone contains the plane spanned by $\left(Z^{\prime}\right)_{\text {red }}$. We may assume $\left(Z^{\prime}\right)_{\text {red }} \cap D^{\prime}=\emptyset$. Hence a general $T^{\prime} \in\left|\mathcal{I}_{Z^{\prime}}(2)\right|$, the divisor $T^{\prime} \cap D$ of $D$ has only two singular points. Hence for a general $T^{\prime} \in\left|\mathcal{I}_{Z^{\prime}}(2)\right|$ and a general hyperplane section $D^{\prime}$ of $X$ the divisor $\left(T^{\prime} \cup D^{\prime}\right) \cap D$ of $D$ is not the double of a rational normal curve, a contradiction. Now assume $E \neq \emptyset$. Since $\operatorname{deg}(H)=6$, then $\Sigma$ is either a line or a conic. In both cases the family of all possible $\Sigma^{\prime}$ does not contain 5 general points of $D$ (at most 3), a contradiction.

Now assume $x=4$. We know that $h^{0}\left(\mathcal{I}_{E_{4,5}}(3)\right) \geq 1$ and we need to prove that $h^{0}\left(\mathcal{I}_{E_{4,5}}(3)\right)=1$, i.e. $h^{1}\left(\mathcal{I}_{E_{4,5}}(3)\right)=2$. Assume $h^{0}\left(\mathcal{I}_{E_{4,5}}(3)\right) \geq 2$. Let $A^{\prime} \subset$ $X$ be a general union of 42 -points and let $V^{\prime} \subset H^{0}\left(D, \mathcal{O}_{D}(3)\right)$ be the image of the restriction map $H^{0}\left(\mathcal{I}_{A^{\prime}}(3)\right) \rightarrow H^{0}\left(D, \mathcal{O}_{D}(3)\right)$. Since $h^{0}\left(\mathcal{I}_{A^{\prime}}(2)\right)=1$ and $h^{1}\left(\mathcal{I}_{A^{\prime}}(3)\right)=0$, we have $\operatorname{dim}\left(V^{\prime}\right)=15$. Since we assumed that $h^{0}\left(\mathcal{I}_{E_{4,5}}(3)\right) \geq 2$, we have $V(-2 B) \neq 0$ for a general $B \subset D$ such that $\sharp(B)=5$. Let $H$ be the zero-locus of a general In characteristic zero a general 2-point of an integral 
variety of positive dimension gives at least two independent conditions to any non-constant linear system $([7])$. Hence $h^{0}\left(\mathcal{I}_{E_{z, 5}}(3)\right)=0$ for all $z \geq 5$, even if $D$ is a quadric cone.

(b2) Assume $k=4$. We have $52 \leq 4 x+3 y \leq 58$ and $3 y+3 f+5=25$. Hence $g=1$ and $y+f=8$. Since $4 x+3 y=4 x+24-3 f \geq 52$, we have $4(x-f) \geq$ 28 and hence $x-f \geq 7$. Hence $x-f-g \geq 6$. Therefore $h^{0}\left(\mathcal{I}_{A}(2)\right)=0$. It is sufficient to check that either $h^{0}\left(\mathcal{I}_{A \cup\{2 O, D\}}(3)\right)=0$ or $h^{1}\left(\mathcal{I}_{A \cup\{2 O, D\}}(3)\right)=0$. This is true, because no case with $y=1$ is an exceptional case when $k=3$ (step. (b2)).

(b3) Assume $k \geq 5$. Since $k-2 \geq 3$, either $h^{0}\left(\mathcal{I}_{A}(k-2)\right)=0$ or $h^{1}\left(\mathcal{I}_{A}(k-2)\right)=0([3])$. In the latter case we have $h^{0}\left(\mathcal{I}_{A}(k-2)\right)=h^{0}\left(\mathcal{I}_{A}(k-\right.$ $1))-k^{2}=h^{0}\left(\mathcal{I}_{A \cup\left\{2 S^{\prime \prime}, D\right\}}(k-1)\right)-k^{2}+3 g$. Hence it is sufficient to check that $f+3 g \leq k^{2}$. This is true, because $3 f+g \leq(k+1)^{2}, g \leq 2$, and $k \geq 5$.

\section{Acknowledgements}

The author was partially supported by MIUR and GNSAGA of INdAM (Italy).

\section{References}

[1] B. Ådlandsvik, Joins and higher secant varieties, Math. Scand. 61 (1987), 213-222.

[2] E. Arbarello and M. Cornalba, Footnotes to a paper of Beniamino Segre, Math. Ann. 256 (1981), 341-362.

[3] E. Ballico, On the defectivity of the complete embeddings of a smooth 3-dimensional quadric. Adv. Geom. 12 (2012), no. 3, 501-508.

[4] J. Alexander and A. Hirschowitz, Un lemme d'Horace différentiel: application aux singularité hyperquartiques de $\mathbb{P}^{5}$, J. Algebraic Geom. 1 (1992), 411-426.

[5] J. Alexander and A. Hirschowitz, An asymptotic vanishing theorem for generic unions of multiple points, Invent. Math. 140 (2000), 303-325.

[6] K. Chandler, A brief proof of a maximal rank theorem for generic 2-points in projective space. Trans. Amer. Math. Soc. 353 (2000), no. 5, 1907-1920. 
[7] L. Chiantini and C. Ciliberto, Weakly defective varieties, Trans. Amer. Math. Soc. 454 (2002), no. 1, 151-178.

[8] C. Ciliberto and R. Miranda, Interpolations on curvilinear schemes, J. Algebra 203 (1998), no. 2, 677-678.

[9] R. Hartshorne, Algebraic Geometry, Springer-Verlag, Berlin-HeidelbergNew York, 1977.

[10] A. Laface, On linear systems of curves on rational scrolls, Geom. Dedicata 90 (2002), 127-144. 
